\title{
Prakash and Sertel's theory of non-cooperative equilibria in social systems - twenty years later
}

\author{
Erdem Başçı ${ }^{a}$, Murat R. Sertel ${ }^{b, c, *}$ \\ "Department of Economics, Bilkent University, Ankara TR-06533, Turkey \\ ${ }^{b}$ Department of Economics, Bog̃aziçi University, Istanbul TR - 80815, Turkey \\ 'Turkish Academy of Sciences, Atatürk Blv. 22I, Ankara TR-06100, Turkey \\ Submitted January 1995, Accepted January 1995
}

\begin{abstract}
This paper is intended as a companion to the paper 'Existence of non-cooperative equilibria in social systems by Prakash and Sertel (1974b) appearing in this volume. It aims to perform two tasks: (1) to give the reader a glimpse at the literature relevant to the existence of equilibria in social systems as it has developed since the writing of the Prakash and Sertel (PS) paper; and (2) to provide a class of examples illustrating where the PS notions of a social system and the non-cooperative equilibrium of a social system generalize the well-known concepts of games, abstract economies and their associated equilibria, showing how the existence theory of PS even today bears economic results beyond where alternative theories are applicable.
\end{abstract}

JEL classification: C62; D51; D62; O30

Keywords: Equilibrium existence; Production economies; Externalities; Technological change; Sustainable Walrasian equilibria

\section{Introduction}

The Prakash and Sertel (1974b) (henceforth PS) paper, 'Existence of non-cooperative equilibria in social systems', which gave rise to the present paper, has

\footnotetext{
* Corresponding author.
} 
remained unpublished for over two decades, coming to the attention of very few theorists ${ }^{1}$ during this period.

Its results in economic theory or game theory had actually formed the main motivation for a certain amount of "background" work by Prakash and Sertel $(1971,1974 a)$ in topological semigroups in the early 1970 s. In this work they had formulated their topological semivector spaces, developing a fixed point theory for these spaces, all with the direct aim of proving their equilibrium existence theorems (Sertel, 1971; Prakash, 1971) relevant to generalized games and economies. In fact, the mathematical basis of the PS (1974b) paper, causing the present paper, can be found in Prakash and Sertel (1974a), followed by their embedding theory in Prakash and Sertel (1976).

A canonical example of a topological semivector space is the collection $\mathscr{K} Q[L]$ of compact and convex non-empty subsets of a real topological vector space (tvs) $L$, where addition is $A+B=\{(a+b) \mid a \in A, b \in B\}$ and scalar multiplication is again as one would expect: $\lambda A=\{\lambda a \mid a \in A\}(A, B \in \mathscr{K} \mathscr{Q}[L]$, $\lambda \in \mathfrak{R})$. Note that $\mathscr{K} \mathscr{Q}[L]$ is the typical space of "feasible regions" in optimization, games, pseudo-games, economies, etc. Observe that the inverse under + does not exist, e.g. for $L=\Re$ and $A=[0,1]$ (The identity element is $\{\underline{0}\}$, where $\underline{0}$ is the "origin" of $L$, and there is no $B \in \mathscr{R Q}[L]$ such that $A+B=\{\underline{0}\}$ or $B+A=\{\underline{0}\}$.) Thus, we have not only a semigroup under + , but in fact a semi vector space under + and scalar multiplication (as defined above). The topological semivector spaces developed in Prakash and Sertel (1974a) are abstracted from $\mathscr{R Q}[L]$, motivated by the importance of this space for economics and game theory. As to Prakash and Sertel (1976), it invents an inverse for each element of a "hyperspace" (collection of subsets) of a tvs, as exemplified by $\mathscr{K} \mathscr{Q}[L]$, and this in a way that jives with the algebra of the semivector space and the continuity of its operations, extending the topological semivector space $\mathscr{K} \mathscr{Q}[L]$ to a topological vector space. Indeed, Prakash and Sertel thus embed $\mathscr{R} Q L]$ in a tvs $\hat{L}$ whenever $L$ is a tvs, choosing $\hat{L}$ locally convex if $L$ is so, etc.

Prakash and Sertel's (1974a) fixed point theory (FPT) of "locally convex" topological semivector spaces generalized the then known FPT in locally convex topological vector spaces (the FPT of Brouwer, 1912; Kakutani, 1941; Tychonoff, 1935; Fan, 1952 and Glicksberg, 1952), and so they had a relatively easy time establishing the existence of equilibria in their (1974b) paper (PS). (This is something that could not have been done, one should emphasize, by use of the Fan and Glicksberg FPT, at least directly, without necessarily invoking both papers of Prakash and Sertel, 1974a, 1976.) In the equilibrium existence theory of PS, we

\footnotetext{
${ }^{1}$ Notably, Ichiishi (1983, p. 74) has remarked:

"Prakash and Sertel (1974) generalized the abstract economy to the situation in which the utility and also the feasible set of a player depend not only on the others' choices of strategy but also on the others' feasible strategy sets (e.g. on enemy's capabilities). Their logic for the existence theorem goes deep (beyond fixed point theorems for topological vector spaces)."
} 
have a very broad and free "endogeneity", in that feasible regions and preferences as well as the behavior of agents, are endogenous, the adjustment process allowing just about everything to depend on everything else: agents' preferences are about not only the joint behavior of all agents (the usual thing), but also the feasible regions of all agents; so, the best responses of agents depend on all the mentioned, witnessing a broad class of externalities in preferences. Furthermore, an agent's preferences are about not just the "current" behavior of all agents, but also pairs of "last" and "current" such behaviors. So, multistage settings are abstractly included to this extent. On the other hand, the feasible regions of all agents depend through a "feasibility map" on the joint behavior of all agents and on themselves, in the sense that a "new" feasible region listing for all agents is a deformation of the "old" such listing as affected by the agents' choice behavior, and the manner in which an individual agent's feasible region is altered may depend, not only on the original feasible region of that agent, but on those of all other agents as well. Thus, we see a rather broad class of externalities in "production" (feasibility) incorporated.

This paper is organized as follows. The next section discusses the literature since 1974 on abstract economies, generalized games, economies and their equilibria. Section 3 provides a class of examples of production economies in which the PS approach has a distinct advantage in establishing the existence of equilibria. Section 4 discusses when the non-cooperative equilibria of these economies will also be sustainable Walrasian equilibria and where (e.g. in the presence of switching costs) they may fail the requisite material balances condition. Section 5 ends the paper with some closing remarks.

\section{A review of the literature}

The construct of an abstract economy and its social equilibrium, introduced by the classical paper of Debreu (1952), differ from a normal form game and its Cournot-Nash equilibrium in that the feasible sets of the players in an abstract economy are allowed to depend on the actions of others. The social equilibrium, whose existence Debreu (1952) proves, involves a finite number of agents, each with a strategy space lying in $\mathfrak{R}^{\mathrm{n}}$. This construct, besides being of interest in its own right for the purpose of modelling social and economic phenomena, has been a valuable tool in proving the existence of competitive equilibria of economies. The first demonstration of this is in the celebrated Arrow and Debreu (1954) paper.

Several extensions of Debreu's (1952) result have appeared in the literature. Mas-Colell (1974) demonstrated, with a novel method differing from that of Arrow and Debreu (1954), that the preferences of consumers need not be transitive or complete for the existence of a competitive equilibrium in finite economies. Shafer and Sonnenschein (1975), inspired by this work of Mas-Colell (1974), and 
using Kakutani's (1941) fixed point theorem, showed that the preferences of agents need not be transitive or complete for the existence of a social equilibrium in an abstract economy. Borglin and Keiding (1976), using an argument concerning the existence of maximal elements, slightly generalized the Shafer and Sonnenschein result by weakening their requirements on preferences a bit further, keeping, however, both the number of agents and the dimensionality of their strategy spaces finite.

Another line of research aimed at relaxing the finiteness condition for the dimensionality of strategy spaces and the number of agents. Yannelis and Prabhakar (1983), relying on a continuous selection theorem of Michael (1956), allowed the strategy sets of agents to lie in any metrizable, locally convex topological vector space, permitting the set of agents to be countably infinite. Toussaint (1984) proved an equilibrium existence theorem for an arbitrary set of agents with strategy sets lying in an arbitrary topological vector space. Toussaint's method of proof, again (cf. Borglin and Keiding, 1976) based on the existence of maximal elements, used the Browder (1968) fixed point theorem. Khan (1985) surveyed much of this and related work. In all of the above, the maps determining feasible regions were assumed continuous. Tulcea (1988) dropped the lower semicontinuity assumption on these maps. Tarafdar (1991) provided a similar result for Hausdorff topological vector spaces and an arbitrary set of agents. After the important steps of Yannelis and Prabhakar (1983) and Toussaint (1984) in dropping the finiteness assumptions on the set of agents and the dimensionality of stratcgy spaces, researchers such as Tan and Yuan (1994) also made advances in relaxing certain restrictions on "better than" and "feasible set" correspondences.

A third line of research modeled the set of agents as a measure space, defining abstract economies and their social equilibria accordingly. Khan and Vohra (1984) accomplished this for finite-dimensional strategy spaces, while Yannelis (1987) as well as Kim et al. (1989) allowed strategy spaces to be infinite-dimensional. All three studies used the Fan (1952)-Glicksberg (1952) fixed point theorem. PS does not take the measure theoretic modeling approach.

All these studies, with minor modifications, adhere to the construct of an abstract economy. In contrast, PS introduces a novel object of a social system. In addition to the dependence of utilities and strategy sets on the strategies chosen by all agents, a social system also allows for the dependence of utilities and strategy sets on the strategy sets of all agents. Moreover, a social system can incorporate phenomena such as addiction or stickiness in behavior (as, perhaps, due to switching costs). The potential applications of such a construct are numerous, as illustrated by the class of examples presented in the following section. Alternative results on abstract economies or generalized games seem to be less well-suited to be applied to such examples.

PS also proves the existence of a non-cooperative equilibrium of such a social system. The existence theorem places no restrictions on the cardinality of the set of agents, and the strategy sets may lie in any locally convex Hausdoff topological 
vector space (cf. Yannelis and Prabhakar, 1983, and Toussaint, 1984). The proof is based on one of the Prakash and Sertel (1974a) fixed point theorems in topological semivector spaces.

Turning now specifically to the topic of competitive equilibrium, Arrow and Debreu (1954) used an abstract economy construct in proving their existence theorem for such equilibria. Thereafter, their method was frequently applied. Examples of its application in finite-dimensional and finite-agent settings can be found in Shafer and Sonnenschein (1975) and Shafer (1976). Toussaint (1984) on the other hand, gave an outstanding example, demonstrating how to use the Arrow and Debreu approach when commodity space is infinite dimensional. This paper also generalizes Bewley's (1972) equilibrium existence result (see below) to the case where preferences need not be complete or transitive.

Three other proof strategies in infinite-dimensional commodity spaces are worth mentioning. One approach is based on market equilibrium, or the so called Gale-Nikaido-Debreu lemma. Infinite-dimensional extensions of this lemma are provided by Florenzano (1983) and Yannelis (1985), and these are applied by Florenzano (1983) and Khan and Yannelis (1991b), respectively, in proving the existence of competitive equilibria. A second approach has its roots in a paper of Negishi (1960), where the existence of competitive equilibria is established by the use of results from welfare economics. Magill (1981) and Mas-Colell (1986) provided infinite-dimensional applications of this approach. A third approach is the, by now, classical one of Bewley (1972), in $\mathscr{L}_{x}$ commodity spaces, based on a limit argument that makes use of existence results in the finite-dimensional case. Khan (1984), Duffie (1986), Yannelis and Zame (1986) are prominent applications of this method. ${ }^{2}$

Before passing on to the next section we want to draw attention to the PS, Lemma 7 (see also Sertel, 1971, Section 3.1.4 and Theorem 3.3.1) on the upper semicontinuity of optimization. This is a tidy generalization of a similar result often attributed to Berge (1959), a yet more specific form of which was shown by Debreu (1952, p. 889). The PS optimization lemma directly states that the set of optimal solutions according to a closed complete preorder on a compact space is upper semicontinuous in the feasible region on which the optimization takes place. Their proof of this result, whose typical economic application concerns the upper semicontinuity of demand, is quite compact.

The next section presents a class of examples in a private ownership economy with finitely many agents and just two commodities. They display several sorts of technological externalities, and preference externalities. Consumers may exhibit addiction or flip-flop tastes, and firms may experience switching costs in

\footnotetext{
${ }^{2}$ Khan and Yannelis (1991a) provides a more complete list of references on the subject, together with a collection of some recent, as well as some older but previously unpublished, very noteworthy work.
} 
production. The question is the existence of a sustainable Walrasian equilibrium, and to answer this question here, we need the results of PS.

\section{A class of examples}

As an economic application of the PS existence theorem for a non-cooperative equilibrium, in this section we will present a class of genuinely economic examples of social systems that satisfy the PS sufficient conditions and hence possess non-cooperative equilibria of economic interest. We will keep technicalities to a minimum, restricting attention to Euclidean space as our underlying tvs and, in fact, to a world of two commodities, for instance, but will try to incorporate in our described social systems a rich variety of interactive aspects, which are normally avoided as troublesome in equilibrium theory.

To this end, we first recall some notation of PS, to which we will henceforth adhere. For any topological space $Y, \mathscr{K}[Y]$ will denote the space of the non-empty compact subsets of $Y$, and when $Y$ lies in a real vector space, $\mathscr{R} \mathscr{Q}[Y]$ will denote the space of the compact and convex non-empty subsets of $Y$.

It will be recalled that, using the terminology and notation of PS, the PS sufficient conditions for the existence of a non-cooperative equilibrium of a social system,

$$
S=\left\{\left(X_{\alpha}, F_{\alpha}, \leqslant_{\alpha}, \delta_{\alpha}\right)_{A}\right\},
$$

are that

(PS1) $X_{\alpha}$ be (non-empty) compact and convex in a locally convex Hausdorff topological vector space.

(PS2) $\mathscr{F}_{\alpha} \subset \mathscr{K} \mathscr{Q}\left[X_{\alpha}\right]$ be a closed and convex cover of $X_{\alpha}$ when $\mathscr{K}\left[X_{\alpha}\right]$ carries the finite topology. ${ }^{3}$

(PS3) $\leqslant_{\alpha}$ be closed as a subset of $\left(X \times \mathscr{F} \times X_{\alpha}\right)^{2}$, as well as upper semiconvex on $X_{\alpha}$.

(PS4) $\delta_{\alpha}: X \times \mathscr{F} \rightarrow \mathscr{K} \mathscr{Q}\left[\mathscr{F}_{\alpha}\right]$ be upper semicontinuous.

Throughout, we choose every $X_{\alpha} \neq \emptyset$ to be compact and convex in $\mathfrak{H}^{2}$, directly satisfying condition (PS1). Condition (PS3) will also be satisfied quite directly, for in each of our examples we will take each $\leqslant_{\alpha}$ to be represented by a real-valued function, which we check to be continuous on $X \times \mathscr{F} \times X_{\alpha}$ as well as

\footnotetext{
${ }^{3}$ For the finite topology see Appendix A.1.1 of PS, or directly see Michael (1951).
} 
quasi-concave on $X_{\alpha}$. For the rest, we will check (PS2) and (PS4) for each example as we proceed.

The social systems in our examples will be allowed to exhibit several types of unusually interactive features. One of these will be in the form of technological externalities in the sense that technologies of firms will be allowed to be affected by each other and/or by productive activities, be these one's own or others'. Next, the profit functions of producers will be allowed to suffer switching costs. Likewise, consumers will be allowed to have preferences dependent on past behavior - be this the consumer's own or others' - and cven on the feasibility (budget set) available to the consumer himself, or on those available to others.

Consider an economy consisting of a sequence of spot markets in each of which, myopically, the consumers are concerned only with maximizing current utility and the firms with maximizing current profit. ${ }^{4}$ The question then is the existence of a sustainable competitive equilibrium for such an economy: does there exist a list of technologies, budget sets, prices, production and consumption plans that can stay at rest while firms maximize profits, consumers maximize utility and markets clear (in the sense that there is no excess demand)? In considering this, one can also allow for the usual kinds of consumption and production externalities, which can be easily handled in the abstract economy framework of Debreu (1952). Among the extensions of Debreu's existence theorem discussed in the previous section, however, there is no work, to our knowledge, whose construct of an abstract economy is rich enough to allow for the presence of the unusual features mentioned above and to be exemplified bclow.

To avoid introducing new terminology, we present our examples in the language of a social system, resorting also to Arrow and Debreu (1952)'s "auctioneer", a fictitious player who sets prices with the value of excess demand as the objective function. (As a result, at any non-cooperative equilibrium of our social system, aggregate excess demand will be non-positive.)

We take our set $A$ of individuals to be finite and partitioned into $\{\{0\}, I, J\}$, where 0 is our "auctioneer", $I$ is the set of consumers, and $J$ is the set of firms. For our social system $S=\left\{\left(X_{\alpha}, \mathscr{F}_{\alpha}, \leqslant_{\alpha}, \delta_{\alpha}\right)_{A}\right\}$, we first describe the characteristics of the firms $j \in J$.

\footnotetext{
${ }^{4}$ To keep the exposition simple, here we do not allow for the presence of assets or futures markets that give trading opportunities over time. These could be incorporated without much difficulty. For instance there could, on top of apples $x$ and bananas $y$, be money $z$ too, and in each period $z$ could only be spent or saved for the next decision moment. A consumer $i \in I$ would then have initial endowments $\left(\bar{x}_{i}^{\prime}, \bar{y}_{i}^{\prime}, \bar{z}_{i}^{\prime}\right)$ in each period $t=1,2, \ldots$, where the money endowment $\bar{z}_{i}^{\prime}=z_{i}^{t-1}$ is the chosen level of non-negative saving in the previous period, the consumption decision at $t$ being taken so as to maximize $u_{i}\left(x_{i}^{\prime}, y_{i}^{\prime}, z_{i}^{\prime}\right)$ subject to $p^{\prime} x_{i}^{\prime}+q^{\prime} y_{i}^{\prime}+z_{i}^{\prime} \leq p^{\prime} \bar{x}_{i}^{\prime}+q^{\prime} \bar{y}_{i}^{\prime}+\bar{z}_{i}^{\prime}$ and $\bar{z}_{i}^{\prime}=z_{i}^{\prime-1}$ ( $t=$ $1,2, \ldots), p^{\prime}$ and $q^{\prime}$ standing for the prices of $x^{\prime}$ and $y^{\prime}$, respectively. Thus, in this model, $z$ is also numeraire, and there is no borrowing from the future. (It might be instructive to think of $x$ (or $y$ ) as labor here.)
} 


\subsection{Firms (producers)}

Taking some $c>0$ sufficiently large, for each firm $j \in J$, we let

$$
X_{j}=\left\{y \in \mathfrak{R}^{2} \mid\|y\| \leq c\right\},
$$

be the behavior space of $j$. Agreeing to give the finite topology 5 to all hyperspaces of a Euclidean space, we take

$$
\mathscr{F}_{j}=\left\{Y \in \mathscr{K} Q\left[X_{j}\right] \underline{0} \in Y\right\}
$$

as the feasibility space of the typical firm $j \in J$. Thus, $\mathscr{F}_{j}$ covers $X_{j}$, and is convex. We also check that $\mathscr{F}_{j}$ is a closed subspace of $\mathscr{K}\left[X_{j}\right]$, the (hyper)space of the non-empty compact subsets of $X_{j}$. To that end,first we note that $G=\{K \in$ $\left.\mathscr{K}\left[X_{j}\right] \mid \underline{0} \in K\right\}$ is closed in $\mathscr{K}\left[X_{j}\right]$ (for, taking $\left.U=X_{j} \backslash \underline{0}\right\}, U \subset X_{j}$ is open, and $\left\{K \in \mathscr{K}\left[X_{j}\right] \mid K \subset U\right\}=G^{c}$ is therefore open in the upper semifinite topology). Since $\mathscr{K} \mathscr{Q}\left[X_{j}\right]$ is closed by A.1, it follows that $\mathscr{F}_{j}=G \cap \mathscr{K} \mathscr{Q}\left[X_{j}\right]$ is also closed in $\mathscr{K}\left[X_{j}\right]$, satisfying (PS2).

The preference $\leqslant_{j}$ of any firm is that represented by its profit function $\pi_{j}: X \times \mathscr{F} \times X_{j} \rightarrow \mathfrak{R}$, and $\pi_{j}$ may be of various forms. One form is that of

$$
\pi_{j}\left(x, F, z_{j}\right)=x_{0} \cdot z_{j}
$$

the inner product of the price $x_{0}$ (chosen by the auctioneer, 0 ) with the production plan $z_{j}$ of firm $j$. Alternatively, we can incorporate switching $\operatorname{costs}^{6}$ in $\pi_{j}$ by giving it a form:

$$
\pi_{j}\left(x, F, z_{j}\right)=x_{01} \min \left\{x_{j 1}, z_{j 1}\right\}+x_{02} z_{j 2} .
$$

Here $\left(x_{01}, x_{02}\right)$ is the price vector chosen by the auctioneer. This reflects switching costs by penalizing period-to-period decreases in absolute quantities of inputs and increases in quantities of outputs. In either form (2) or (3), $\pi_{j}$ is readily verified to be a continuous function (so that $\preccurlyeq_{j}$ is closed), and it can easily be checked to be quasi-concave in $z_{j}$ (so that $\preccurlyeq_{j}$ is upper semiconvex on $X_{j}$ ). Thus (PS3) is satisfied by $\leqslant$, here.

Regarding the feasibility maps:

$$
\delta_{j}: X \times \mathscr{F} \rightarrow\left[\mathscr{F}_{j}\right]
$$

one can imagine four interesting possibilities. The first possibility is

$$
\delta_{j}(x, F)=\left\{\rho\left(x_{j}\right) F_{j}\right\},
$$

\footnotetext{
${ }^{5}$ See footnote 3 .

${ }^{6}$ Switching costs have often been recognized as an important factor affecting economic behavior. A most recent example (although in a different context) is in the work of Banks and Sundaram (1994): "... Indeed, it is difficult to imagine a relevant economic decision problem in which the decision maker may costlessly move between altematives"' (Banks and Sundaram, 1994, p. 687).
} 
where the function $\rho$ determines a real number $\rho\left(x_{j}\right) \in[0,1]$ for each production plan $x_{j}$ of firm $j \in J$. Thus, as a function of productive activity the production set is allowed to shrink but not allowed to swell. An example is where

$$
\rho\left(x_{j}\right)=1-\frac{\left\|x_{j}\right\|}{2 c} .
$$

This captures the idea of depreciation by use or a sort of depletion. As a second possibility, the production plan of a firm may affect the productivity of the other firms. For instance, if the production of firm $j^{\prime} \in J$ is detrimental to the technology of firm $j \in J$, we may see the effect of this through a feasibility map $\delta_{j}$ of the form:

$$
\delta_{j}(x, F)=\left\{\rho\left(x_{j^{\prime}}\right) F_{j}\right\},
$$

where $\rho: X_{j^{\prime}} \rightarrow[0,1]$ is as above. If, instead, $\rho_{j}$ is a function that typically takes values in excess of unity on $X_{j}$, and we write

$$
\delta_{j}(x, F)=\left\{\left(\rho_{j}\left(x_{j}\right) F_{j}\right) \cap X_{j}\right\} .
$$

This will reflect a certain type of learning by doing, which will preserve constant-returns-to-scale technologies. For a special learning-by-doing effect that favors small experiments in production, but regards large production as depletionary, one may regard the functional form:

$$
\rho_{j}\left(x_{j}\right)=\left\|x_{j}\right\|^{1 / 2} \text {. }
$$

Assuming that $\rho$ is continuous, $\delta_{j}$ will be (upper semi-)continuous in both forms (4) and (5), satisfying (PS4).

As a next possibility, firm $j \in J$ can learn parts of the technology of firm $j^{\prime} \in J$ and combine these with its own know-how, to obtain its next period's technology. ${ }^{7}$ One such type of technology spill-over can be modeled by the feasibility map:

$$
\delta_{j}(x, F)=\left\{h\left(F_{j} \cup F_{j^{\prime}}\right)\right\},
$$

where $h: \mathscr{K}\left[\mathfrak{H}^{2}\right] \rightarrow \mathscr{K}\left[\mathfrak{H}^{2}\right]$ is the (closed) convex hull operator. Now factorization $F \mapsto\left\{F_{\alpha}\right\}_{\alpha \in A}$ is continuous, by Prakash and Sertel (1977), and the union operator is continuous on $\mathscr{K}\left[\Re^{2}\right] \times \mathscr{K}\left[\Re^{2}\right]$. Since projection $\left\{F_{\alpha}\right\}_{\alpha \in A} \rightarrow\left(F_{j}, F_{j}\right)$ is also continuous, $F_{j} \cup F_{j^{\prime}}$ is continuous in $F$, hence in $(x, F)$. By Sertel (1989), furthermore, the closed convex hull operator $h$ is continuous on $\mathscr{K}^{[}\left[\mathfrak{R}^{m}\right]$. Thus, $\delta_{j}$ in (6) is also (upper semi-)continuous, again satisfying (PS4).

\footnotetext{
The firm $j \in J$ in (6) and (7) below can be seen as a bartender who always knows how to make cocktails (convex combinations) of recipes (productions) he (it) knows, in (6) discovering an entire new recipe list $F_{j}$, and in (7) discovering only some new recipe $x_{j}$. Once any recipes are "discovered", their convex combinations with all known (previously discovered) recipes become known too.
} 
The fourth possibility is that firm $j$ sees the production activity $x_{j^{\prime}}$ of firm $j^{\prime}$ and, thus, "learns" all convex combinations of $x_{j}$, with any $x_{j} \in F_{j}$. This sort of "learning from one's neighbors" is expressed by the following form of feasibility map:

$$
\delta_{j}(x, F)=\left\{h\left(\left\{x_{j^{\prime}}\right\} \cup F_{j}\right)\right\} .
$$

This feasibility map is also (upper semi-)continuous, as one can establish by arguments similar to those in the case of (6), once again obeying (PS4).

Now we describe the characteristics of the consumers $(i \in I)$.

\subsection{Consumers}

A consumer $i \in I$ is specified by an ordered quadruplet:

$$
\left(X_{i}, \omega_{i}, \theta_{i}, u_{i}\right) \text {, }
$$

where picking some $d>0$ sufficiently large, we always take the "consumption set" to be

$$
X_{i}=\left\{z \in \mathscr{R}_{+}^{2} \mid z \leq(d, d)\right\},
$$

while the "initial endowment" $\omega_{i} \in X_{i}$ with $\omega_{i} \gg \underline{0}$, and the "portfolio" $\theta_{i} \in \mathfrak{R}_{+}^{J}$ (with $\sum_{k \in I} \theta_{k j}=1$ for each firm $j \in J$ ) are as usual, and the "utility" is some $u_{i}: X \times \mathscr{F} \times X_{i} \rightarrow \mathfrak{R}$, where $\mathscr{F}_{j}$ for $j \in J$ is given by (1), $\mathscr{F}_{i}$ for cach consumer $i \in I$ is given by

$$
\mathscr{F}_{i}=\mathscr{K} Q\left[X_{i}\right] \text {, }
$$

and $\mathscr{F}_{0}=\left\{X_{0}\right\}$ with $X_{0}=\left\{x_{0} \in \mathfrak{R}_{+}^{2} \mid x_{01}+x_{02}=1\right\}$ will be the price simplex for the auctioneer.

To express the idea of a budget set for consumer $i \in I$, we write

$$
B\left(x_{0}, x_{J} ; \omega_{i}, \theta_{i}\right)=\left\{x_{i} \in X_{i} \mid x_{0} \cdot x_{i} \leq x_{0} \cdot \omega_{i}+\max \left\{0, \sum_{j \in J} \theta_{i j} x_{0} \cdot x_{j}\right\}\right\},
$$

where $x_{0}$ is the auctioneer's chosen price vector, $x_{J} \in X_{J}=\prod_{j \in J} X_{j}$ stands for the production plan profile of the producers. Now, clearly,

$$
\mathscr{F}_{i} \supset\left\{B\left(x_{0}, x_{J} ; \omega_{i}, \theta_{i}\right) \mid x_{0} \in X_{0}, x_{J} \in X_{J}\right\},
$$

while $\mathscr{F}_{i}$ satisfies (PS2), since it is closed in $\mathscr{K}\left[X_{i}\right]$ (see A.1), covers $X_{i}$ and is convex.

For the feasibility map $\delta_{i}: X \times \mathscr{F} \rightarrow\left[\mathscr{F}_{i}\right]$ we take

$$
\delta_{i}(x, F)=\left\{B\left(x_{0}, x_{j} ; \omega_{i}, \theta_{i}\right)\right\},
$$


whose unique selection is the budget set map $\beta_{i}: X \times \mathscr{F} \rightarrow \mathscr{F}_{i}$, with

$$
\beta_{i}(x, F)=B\left(x_{0}, x_{j} ; \omega_{i}, \theta_{i}\right)
$$

actually independent of $F$ and $x_{I} \in X_{I}=\prod_{k \in I} X_{k}$, depending only on $\left(x_{0}, x_{J}\right)$. From A.2 we see that $\beta_{i}$ is continuous (since the budget size $b=x_{0} \cdot \omega_{i}+$ $\max \left\{0, \Sigma_{j \in J} \theta_{i j} x_{0} \cdot x_{j}\right\}$ is continuous in $\left\{x_{J}, \omega_{i}, \theta_{i}\right\}$ and hence also $\delta_{i}$ is (upper semi-)continuous, thus satisfying (PS4).

So far, our consumers are quite classical text-book types; what will distinguish them from the run of the mill will be their preferences. Each consumer $i$ 's preference $\preccurlyeq_{i}$ will be represented by the utility $u_{i}: X \times \mathscr{F} \times X_{i} \rightarrow \mathfrak{R}$, and it is in the specification of this utility that we capture certain possible paradigmatic characteristics that our consumers may exhibit. For this purpose, it will suffice to regard the general Cobb-Douglas-inspired form:

$$
u_{i}\left(x, F, z_{i}\right)=z_{i 1}^{\mu_{i}(x, F)} z_{i 2}^{1-\mu_{i}(x, F)},
$$

where, $\mu_{i}: X \times \mathscr{F} \rightarrow[0,1]$ is a continuous function determining the elasticity of utility w.r.t. the consumption of the two goods, $z_{i 1}$ and $z_{i 2}$. The continuity of $\mu_{i}$ ensures that of $u_{i}$, so $\leqslant_{i}$ is always closed. The fact that $\mu_{i}(x, F) \in[0,1]$ ensures that $u_{i}$ is concave in $z_{i}$, so $\preccurlyeq_{i}$ is certainly upper semiconvex on $X_{i}$. Thus (PS3) is satisfied by $\preccurlyeq_{i}$ here.

First, we consider the case of $\mu_{i}$ dependent only on $x$, in particular $\mu_{i}(x, F)$ $=r_{i}(x)$, so that

$$
u_{i}\left(x, F, z_{i}\right)=z_{i 1}^{r_{i}(x)} z_{i 2}^{1-r_{i}(x)},
$$

where $r_{i}$ is continuous. Specifically, setting

$$
r_{i}(x)=\frac{x_{i 1}}{x_{i 1}+x_{i 2}}
$$

exemplifies a paradigm of addiction. The more the consumer $i$ consumes of alcoholic beverages $\left(x_{i 1}\right)$, the more partial he becomes toward them in his next consumption decision $\left(z_{i 1}\right)$. Alternatively, setting

$$
r_{i}(x)=\frac{x_{i 2}}{x_{i 1}+x_{i 2}},
$$

we obtain a "flip-flop" boredom effect, whereby higher consumption of one good renders the consumer more easily bored by this good and tilts him to be more partial towards the other in his next consumption decision.

Furthermore, writing

$$
r_{i^{\prime}}(x)=\frac{x_{i 1}}{x_{i 1}+x_{i 2}},
$$


gives us an emulation effect, where consumer $i^{\prime}$ becomes more partial towards wearing dark suits $x_{i^{\prime} 1}$ the more his boss $i$ wears them $\left(x_{i 1}\right)$. Likewise, writing

$$
r_{i^{\prime}}(x)=\frac{x_{i 2}}{x_{i 1}+x_{i 2}}
$$

gives the opposite effect, which we may call negative emulation where consumer $i^{\prime}$ becomes less partial towards wearing dark suits the more his boss' butler $i$ wears them.

The peculiarities of consumer preferences displayed so far are in the genre of externalities that have certainly been discussed both, verbally (e.g. by Veblen, 1899 , in the case of emulation) and theoretically (e.g. by McKenzie, 1955; ${ }^{8}$ Shafer and Sonnenschein, 1975; von Weizsäcker, 1971), and our indicating to such possibilities heralds no news. The next family of possibilities in preference externalities, however, may not be such old hat in general equilibrium existence theory. For now we take $\mu_{i}$ to be of the form $\mu_{i}(x, F)=s_{i}\left(F_{i}\right)$, dependent on the feasibility $F_{i}$ (alone), where the function $s_{i}: \mathscr{F} \rightarrow[0,1]$ is continuous. Thus, we are now looking at the case where

$$
u_{i}\left(x, F, z_{i}\right)=z_{i 1}^{s_{i}\left(F_{i}\right)} z_{i 2}^{1-s_{i}\left(F_{i}\right)},
$$

in order to model a certain freedom effect. ${ }^{9}$ Consider, first, the specific form

$$
s_{i}\left(F_{i}\right)=\frac{1}{\sqrt{2} d} \sup \left\{\|z\| \mid z \in F_{i}\right\},
$$

where $s_{i}$ measures the sup norm of the consumer's own feasibility $F_{i}$. In this case, the greater the consumer's freedom in choosing $z$ with a large norm, the less his partiality for happiness pills $\left(z_{i 2}\right) .{ }^{10}$ Alternatively, we can take either of

$$
\begin{aligned}
& s_{i}\left(F_{i}\right)=\frac{1}{d^{2}} \sup \left\{z_{1} z_{2} \mid z \in F_{i}\right\}, \\
& s_{i}\left(F_{i}\right)=\frac{1}{d} \max \left\{\sup \left\{z_{1} \mid z \in F_{i}\right\}, \sup \left\{z_{2} \mid z \in F_{i}\right\}\right\},
\end{aligned}
$$

\footnotetext{
${ }^{8}$ The entry on "externalities" by Laffont (1987) in the New Palgrave: A Dictionary of Economics mentions only McKenzie (1955) regarding equilibrium existence results in the presence of externalities.

${ }^{9}$ One should be cautious not to regard this as a Slutsky-like income (or wealth) effect, as the examples below should clearly show.

${ }^{10}$ The reader may find it entertaining to observe and tabulate (as below) demand on budget sets $B=h(\{(0,0),(d, 0),(0, d)\}), \quad B^{\prime}=h(\{(0,0),(d / 2,0),(0, d)\}), \quad \frac{1}{2} B=h(\{(0,0),(d / 2,0),(0, d / 2)\})$, $B_{n}=h(\{(0,0),(d / 2,0),(0, d / n)\}), B_{x}=h(\{(0,0),(d / 2,0),(0,0)\})$.

\begin{tabular}{l|lllll} 
Budget set & $B$ & $B^{\prime}$ & $\frac{1}{2} B$ & $B_{n}$ & $B_{x}$ \\
\hline Demand & $(d, 0)$ & $\left(\frac{d}{2}, 0\right)$ & $\left(\frac{d}{4}, \frac{d}{4}\right)$ & $\left(\frac{d}{4}, \frac{d}{2 n}\right)$ & $B_{x}$
\end{tabular}
}




$$
\begin{aligned}
& s_{i}\left(F_{i}\right)=\frac{1}{d} \min \left\{\sup \left\{z_{1} \mid z \in F_{i}\right\}, \sup \left\{z_{2} \mid z \in F_{i}\right\}\right\}, \\
& s_{i}\left(F_{i}\right)=\frac{1}{d^{2}} \sup \left\{z_{1} \mid z \in F_{i}\right\} \sup \left\{z_{2} \mid z \in F_{i}\right\},
\end{aligned}
$$

as a measure of the consumer's freedom of choice, ${ }^{11}$ his partiality for jolly pills $\left(z_{i 2}\right)$ decreasing with freedom. Likewise, we could let $\mu_{i}(x, F)$ depend instead on other consumers' feasibilities, for instance by setting in each above case $\mu_{i}(x, F)$ $=s_{i}\left(F_{i}^{\prime}\right)$ for some $i^{\prime} \in I \backslash\{i\}$. The cases (9) to (13) above would then have their analogous forms $\left(9^{\prime}\right)$ to $\left(13^{\prime}\right)$ with $F_{i}$ replaced by $F_{i^{\prime}}$.

Of course, various combinations and derivatives of these forms - and certainly yet other forms - can be imagined for the functions $\mu_{i}$. As remarked at the outset of specifying the Cobb-Douglas-like form with elasticities $\mu_{i}$ and $1-\mu_{i}$, all of the above considered forms of externality in preference are admissible from the viewpoint of satisfying the PS sufficient conditions for the existence of equilibrium, since preference $\preccurlyeq_{i}$ in each case is closed and on $X_{i}$ upper semiconvex.

\subsection{The auctioneer}

Finally, regarding our auctioneer $0 \in A$, we specify:

$$
\begin{aligned}
& X_{0}=\left\{p \in \Re_{+}^{2} \mid p_{1}+p_{2}=1\right\}, \\
& \mathscr{F}_{0}=\left\{X_{0}\right\},
\end{aligned}
$$

with $\delta_{0}: X \times \mathscr{F} \rightarrow\left[\mathscr{F}_{0}\right]$ taken as the constant function with value:

$$
\delta_{0}(x, F)=\left\{X_{0}\right\}
$$

The auctioneer's preference is that represented by $u_{0}: X \times \mathscr{F} \times X_{0} \rightarrow \mathscr{R}$, defined through

$$
u_{0}\left(x, F, z_{0}\right)=z_{0} \cdot\left(\sum_{i \in I}\left(x_{i}-\omega_{i}\right)-\sum_{j \in J} x_{j}\right) .
$$

Thus, our auctioneer is quite classical, $\mathscr{F}_{0}$ clearly obeying (PS2), $\preccurlyeq_{0}$ obeying (PS3), and $\delta_{0}$ trivially satisfying (PS4).

\section{Non-cooperative equilibria as sustainable Walrasian equilibria}

We have run through a broad class of examples allowing for several sorts of endogeneity in technologies and various kinds of technological externality. Then,

\footnotetext{
${ }^{11}$ Another possible case one might consider for $s_{i}\left(F_{i}\right)$ is also the normalized Lebesque measure of $F_{i}$ (i.e. the measure divided by $d^{2}$ ). Cf. Kreps (1979), Barberá and Pattanaik (1984), Barberá et al. (1984), Pattanaik and Xu (1990).
} 
again, we have considered over a dozen types of endogeneity in preferences or of preferential externalities. In each case we have checked that the sufficient conditions PS1-PS4 of Prakash and Sertel for the existence of a non-cooperative equilibrium are satisfied. This means that we could take several different types of producer, each subject to one or another of our various technological externalities or switching costs, and we could mix this motley bunch with a yet motlier bunch of consumers, each exhibiting one or another of the various preferential externalities we have discussed, and we would still be guaranteed the existence of non-cooperative equilibria for the social system they comprise. These equilibria would furthermore be genuinely economic equilibria and, at least in the absence of switching costs, Walrasian in the usual sense of satisfying material balances and individual optimization - in the form of profit maximization, subject to production sets for producers and utility maximization subject to the budget constraints of consumers - as well as sustainable in the sense that the endogenous feasibilities are also at rest.

To be more specific, in the absence of switching costs (e.g. when all the firms have profit functions of form (2)), we can show by standard arguments that at any non-cooperative equilibrium $\left(x^{*}, F^{*}\right)$, the material balances condition:

$$
\sum_{i \in I}\left(x_{i}^{*}-\omega_{i}\right)-\sum_{j \in J} x_{j}^{*} \leq 0,
$$

holds. To that end, first we observe that $x_{0}^{*} \cdot x_{j}^{*} \geq 0$ has to hold for each $j \in J$ since $0 \in F_{j}^{*}$. Hence, the feasibility (budget, see (8)) requirement on a consumer's choice can be re-expressed as

$$
x_{0}^{*} \cdot\left(x_{i}^{*}-\omega_{i}\right)-\sum_{j \in J} \theta_{i j} x_{0}^{*} \cdot x_{j}^{*} \leq 0,
$$

for each $i \in I$. Adding this inequality over consumers, we obtain:

$$
x_{0}^{*} \cdot\left(\sum_{i \in I}\left(x_{i}^{*}-\omega_{i}\right)-\sum_{j \in J} x_{j}^{*}\right) \leq 0
$$

which shows that the maximum "utility" of the auctioneer is non-positive. Now, suppose that in some commodity, the aggregate excess demand were positive, permitting the auctioneer higher utility $u_{0}$ than that at the fixed point (by adjusting prices suitably). This would contradict that $u_{0}\left(x^{*}, F^{*}, \cdot\right)$ is maximized on $X_{0}$ at $x_{0}^{*}$, and so we conclude that aggregate excess demand in each commodity is non-positive.

In the presence of switching costs (i.e. when some firms have profit functions of form (3)), however, a non-cooperative equilibrium need not satisfy material balances. To see where the standard argument fails, imagine the possibility of a non-cooperative equilibrium where $x_{1 j}^{*}=-1, x_{2 j}^{*}=0$, and $x_{01}^{*}=1$. Now at equilibrium $z_{j}^{*}=x_{j}^{*}$ and profit $\pi_{j}^{*}=x_{01}^{*} z_{1 j}^{*}+x_{02}^{*} z_{2 j}^{*}<0$, but the consumers' limited liability (see (8)) prevents this loss from being reflected in the typical 
consumer's budget, so consumer $i$ behaves as if the loss of $j$ is not his liability even if he fully owns firm $\mathcal{X}\left(\theta_{i j}=1\right)$ and owns no other stock $\left(\theta_{i k}=0\right.$ for every $k \in J \backslash\{j\})$. But then (15) does not follow from (8). Therefore, with switching costs of the type we have supposed (3), we cannot guarantee that non-cooperative equilibria will also be sustainable Walrasian.

\section{Closing remarks}

To be reader-friendly, and as simple as possible in our exposition, we have kept our examples in the realm of a strip-miner, i.e. we have only scratched the surface of the rich domain of imaginable cases covered by the PS sufficient conditions for the existence of equilibrium, and economists will easily find more examples relevant to their special interests. Where the applications of the PS equilibrium existence theorem may be especially fruitful is in the general area of public economics, abundant in externalities. In particular, environmental economists are welcome to apply the theorem in their domain of discourse, just as researchers in adjustment costs, in sustainable growth, in endogenous growth may find it attractive to take advantage of the variety of externalities allowed by the theorem.

Finally, the facility of dealing with hyperspaces in the PS model may be attractive to researchers in extending preferences to hyperspaces, as in the literature on the ranking of opportunity sets (e.g. Kreps, 1979; Barberá and Pattanaik, 1984; Barberá et al., 1984; Pattanaik and Xu, 1990). It will have been noted that individuals' preferences in the PS social systems already comprise such extensions as their restrictions to appropriate subspaces.

\section{Appendix}

A.l. Theorem: Let $X \neq \emptyset$ be a locally compact space lying in a real Hausdorff topological vector space $L$. Then $\mathscr{H}[X] \subset \mathscr{R}[X]$ is closed, under the finite topology on $\mathscr{K}[X]$.

Proof. Defining $\mathscr{H}=\mathscr{K}[X] \backslash \mathscr{K} \mathscr{Q}[X]$, take any $H \in \mathscr{H}$. We construct an open nbd $\mathscr{U}$ with $H \in \mathscr{U} \subset \mathscr{H}$. To this end, observe that, since $H$ is non-convex, there are points $x, x^{\prime} \in H$ and $\lambda \in[0,1]$ such that $\bar{x}=\lambda x+(1-\lambda) x^{\prime} \notin H$. As convex combination with coefficients $(\lambda, 1-\lambda)$ (i.e. the function $\Lambda: L \times L \rightarrow L$ defined through $\left.\Lambda\left(y, y^{\prime}\right)=\lambda y+(1-\lambda) y^{\prime}\right)$ is continuous, taking any open $\mathrm{nbd} V \subset X \backslash H$ of $\bar{x}$, there are open nbds $U$ and $U^{\prime}$ of $x$ and $x^{\prime}$, resp., such that $\lambda y+(1-\lambda) y^{\prime}$ $\in V$ whenever $y \in U$ and $y^{\prime} \in U^{\prime}$ (i.e. $A\left(U \times U^{\prime}\right) \subset V$ ). As $L$ (hence $X \subset L$ ) is Hausdorff and $X$ is locally compact, w.l.o.g., $V$ may be assumed compact. Thus, $V^{c}=X \backslash V$ is an open nbd of $H$. Now writing $\mathscr{U}=\langle U\rangle_{-} \cap\left\langle U^{\prime}\right\rangle_{-} \cap\left\langle V^{c}\right\rangle_{+}$, where $\langle U\rangle_{-}=\{K \in \mathscr{K}[X] \mid K \cap U \neq \emptyset\},\left\langle U^{\prime}\right\rangle_{-}=\left\{K \in \mathscr{K}[X] \mid K \cap U^{\prime} \neq \emptyset\right\}$ and 
$\left\langle V^{c}\right\rangle_{+}=\left\{K \in \mathscr{H}[X] \mid K \subset V^{c}\right\}$, we have $H \in \mathscr{U} \subset \mathscr{H}$, while $\langle U\rangle_{-}$and $\left\langle U^{\prime}\right\rangle_{-}$ are sub-basic open sets in the lower semifinite topology and $\left\langle V^{c}\right\rangle_{+}$is a basic open set in the upper semifinite topology on $\mathscr{K}[X]$, so $\mathscr{U}$ is open in the finite topology (i.e. the coarsest topology containing both the upper and the lower semifinite topologies) on $\mathscr{H}[X]$. Thus, $\mathscr{H}$ is open, i.e. $\mathscr{K} \mathscr{Q}[X]$ is closed in $\mathscr{K}[X]$, as to be shown.

A.2. Continuity of budget correspondences. Proving the continuity of the "budget correspondences" $B_{i}:(p, b) \rightarrow B_{i}(p, b)=\left\{x_{i} \in X_{i} \mid p \cdot x_{i} \leq b\right\}$ on $X_{0} \times(0, \infty)$, where $X_{0}$ is the price simplex (here in $\Re_{+}^{2}$ ), is by now a standard exercise for economists. ${ }^{12}$ Nevertheless, we would like to give a direct proof.

Using A.1.5 of PS, we first establish the upper semicontinuity of $B_{i}$ by showing that the graph $G\left(B_{i}\right)=\left\{(p, b, x) \in X_{0} \times(0, \infty) \times X_{i} \mid p \cdot x_{i} \leq b\right\}$ of $B_{i}$ is closed. For this, simply note that the "deficit" $v\left(p, b, x_{i}\right)=p \cdot x_{i}-b$ is continuous and that $G\left(B_{i}\right)=v^{-1}((-\infty, 0])$ is, therefore, closed.

To establish the lower semicontinuity of $B_{i}$, we take any open $V \subset X_{i}$ and show that the inverse image $B_{i}^{-1}\left(\langle V\rangle_{-}\right)$of the (sub-basic) open set $\langle V\rangle_{-}$(of the l.s.f. topology) is open. To that end, take any $(\bar{p}, \bar{b}) \in B_{i}^{-1}\left(\langle V\rangle_{-}\right)$. Thus, $B_{i}(\bar{p}, \bar{b}) \cap$ $V \neq 0$ and, since $b>0$, there exists $\bar{x} \in V$ such that $\bar{p} \cdot \bar{x}-\bar{b}<0$. Since the deficit function $v$ is continuous, there exists a nbd $U$ of $(\bar{p}, \bar{b})$ such that for all $(p, b) \in U, v(U \times\{\bar{x}\}) \subset(-\infty, 0)$, so that $\bar{x} \in B_{i}(p, b)$ and hence $B_{i}(U) \subset$ $\langle V\rangle_{-}$. Thus, $B_{i}^{-1}\left(\langle V\rangle_{-}\right)$is open, completing our proof.

\section{References}

Arrow, K.J. and G. Debreu, 1954, Existence of an equilibrium for a competitive economy, Econometrica $22,265-290$.

Banks, J.S. and R.K. Sundaram, 1994, Switching costs and the Gittins index, Econometrica 3, 687-694.

Barberá, S. and P.K. Pattanaik, 1984, Extending an order on a set to the power set: Some remarks on Kannai and Peleg's approach, Journal of Economic Theory 32, 185-191.

Barberá, S., C.R. Barret and P.K. Pattanaik, 1984, On some axioms for ranking sets of alternatives, Journal of Economic Theory 33, 301-308.

Berge, C., 1959, Espaces topologiques (Dunod, Paris): English translation: 1963, Topological spaces (Oliver and Boyd, Esinburg and London).

Bewley, T., 1972, Existence of equilibrium with infinitely many commodities, Journal of Economic Theory 4, 514-540.

Borglin, A. and H. Keiding, 1976, Existence of equilibrium actions and of equilibrium: A note on the 'new' existence theorems, Journal of Mathematical Economics 3, 313-316.

Brouwer, L.E., 1912, über Abbildungen von Manigfaltigkeiten, Mathemathische Annalen 71, 97-115.

\footnotetext{
${ }^{12}$ In fact, Ichiichi (1983) sets it as Exercise 1 (p. 39), suggesting a proof in several (eight) spelled-out steps.
} 
Browder, F.E., 1968, The fixed point theory of multi-valued mappings in topological vector spaces, Mathemathische Annalen 177, 283-301.

Debreu, G., 1952, A social equilibrium existence theorem, Proceedings of the National Academy of Sciences of the U.S.A. 38, 386-393.

Duffie, D., 1986, Competitive equilibria in general choice spaces, Journal of Mathematical Economics $15,1-23$.

Fan. K., 1952, Fixed point and minimax theorems in locally convex topological linear spaces, Proceedings of the National Academy of Sciences of the U.S.A. 38, 121-126.

Florenzano, M., 1983, On the existence of equilibria in economies with an infinite dimensional commodity space, Joumal of Mathematical Economics 12, 207-219.

Glicksberg, I.L., 1952, A further generalization of the Kakutani fixed point theorem with applications to Nash equilibrium points, Proceedings of the American Mathematical Society 3, 170-174.

Ichiishi, T., 1983, Game theory for economic analysis (Academic Press, New York).

Kakutani, S., 1941, $\Lambda$ generalization of Brouwer's fixed point theorem, Duke Mathematical Journal 8 , $457-459$.

Khan, M.A., 1984, A remark on the existence of equilibria in markets with out ordered preferences and with a Riesz space of commodities, Journal of Mathematical Economics 13, 165-169.

Khan, M.A., 1985, On extensions of the Cournot-Nash theorem, in: C.D. Aliprantis, O. Burkinshaw and N.J. Rothman, eds. Advances in equilibrium theory, (Springer-Verlag, Berlin).

Khan, M.A. and R. Vohra, 1984, Equilibrium in abstract economies without ordered preferences and with a measure space of agents, Journal of Mathematical Economics 13, 133-142.

Khan, M.A. and N.C. Yannelis, eds., 1991a, Equilibrium theory in infinite dimensional spaces (Springer-Verlag, Berlin).

Khan, M.A. and N.C. Yannelis, $1991 \mathrm{~b}$, Equilibria in markets with a continuum of agents and commodities, in: M.A. Khan, and N.C. Yannelis, eds., Equilibrium theory in infinite dimensional spaces (Springer-Verlag, Berlin).

Kim, T., K. Prikry and N.C. Yannelis, 1989, Equilibria in abstract economies with a measure space of agents and with an infinite dimensional strategy space, Journal of Approximation Theory 56, 256-266.

Kreps, D.M., 1979, A representation theorem for preference for flexibility, Econometrica 47, 565-577.

Laffont, J.J., 1987, Externalities, in: J. Eatwell, M. Milgate and P. Newman, eds., The new Palgrave: A dictionary of economics (The Macmillan Press Limited, London).

Magill, M., 1981, An equilibrium existence theorem, Joumal of Mathematical Analysis and Applications 84. 162-169.

Mas-Colell. A., 1974, An equilibrium existence theorem without complete or transitive preference, Joumal of Mathematical Economics 1, 237-246.

Mas-Colell, A., 1986, The price equilibrium existence theorem in topological vector lattices, Econometrica 54, 1039-1053.

McKenzie, L.W., 1955, Competitive equilibrium with dependent consumer preferences, in: H.A. Antosiewicz, ed., Proceedings of the second symposium in linear programming, vol. 1, National Bureau of Standards and Directorate of Management Analysis, DCS/Comptroller, USAF, Washington, $\mathrm{DC}$.

Michael, E., 1951, Topologies on spaces of subsets, Transactions of the American Mathematical Society $71,152-182$.

Michael, E., 1956, Continuous selections I, Annals of Mathematics 63, 361-382.

Negishi, T., 1960, Welfare economics and existence of an equilibrium for a competitive economy, Metroeconomica 12, 92-97.

Pattanaik, P.K. and Y. Xu, 1990, On ranking opportunities in terms of freedom of choice, RecherchesEconomiques-de-Louvain 54, 383-390.

Prakash, P., 1971, Foundations of systems: For decision, planning, control and social/economic analysis, Unpublished Ph.D. disseration, MIT, Cambridge, MA. 
Prakash, P. and M.R. Sertel, 1971, Semilinear (topological) spaces with applications, Proceedings of the Second Florida Symposium on Automata and Semigroups, University of Florida, Gainesville, Florida.

Prakash, P. and M.R. Sertel, 1974a, Topological semivector spaces: Convexity and fixed point theory, Semigroup Forum 9, 117-138.

Prakash, P. and M.R. Sertel, 1974b, Existence of non-cooperative equilibria in social systems, Discussion Paper no. 92, (Revised, 1976). The Center for Mathematical Studies in Mathematics and Management Science, Northwestem University, Evanston, IL.

Prakash, P. and M.R. Sertel, 1976, Hyperspaces of topological vector spaces: Their embedding in topological vector spaces, Proceedings of the American Mathematical Society 61, 163-168.

Prakash, P. and M.R. Sertel, 1977, On the continuity of Cartesian product and factorization, METU Journal of Pure and Applied Sciences 10, 241-264.

Sertel, M.R., 1971, Elements of equilibrium methods for social analysis, Unpublished Ph.D. dissertation, MIT, Cambridge, MA.

Sertel, M.R., 1989, On the continuity of closed convex hull, Mathematical Social Sciences 18, 297-299.

Shafer, W., 1976, Equilibrium in economies without ordered preferences or free disposal, Journal of Mathematical Economics 3, 135-137.

Shafer, W. and H. Sonnenschein, 1975, Equilibrium in abstract economies without ordered preferences, Journal of Mathematical Economics 2, 345-348.

Tan, K.K. and X.Z. Yuan, 1994, Existence of equilibrium for abstract economies, Journal of Mathematical Economics 23, 243-251.

Tarafdar, E., 1991, A fixed point theorem and equilibrium points of an abstract economy, Journal of Mathematical Economics 20, 211-218.

Toussaint, S., 1984, On the existence of equilibria in economies with infinitely many commodities and without ordered preferences, Journal of Economic Theory 33, 98-115.

Tulcea, C.I., 1988, On the approximation of upper-semicontinuous correspondences and the equilibriums of generalized games, Journal of Mathematical Analysis and Applications 136, 267-289.

Tychonoff, A., 1935, Ein Fixpunktsatz, Mathematische Annallen 111, 767-776.

Veblen, T.B., 1899, The theory of the leisure class: An economic study in the evolution of institutions (The Macmillan Company, New York).

von Weizsäcker, C.C., 1971, Notes on endogenous change of tastes, Journal of Economic Theory 3, $345-372$.

Yannelis, N.C., 1985, On a market equilibrium theorem with an infinite number of commodities, Journal of Mathematical Analysis and Applications 108, 595-599.

Yannelis, N.C., 1987, Equilibria in noncooperative models of competition, Journal of Economic Theory 41, 96-111.

Yannelis, N.C. and N.D. Prabhakar, 1983, Existence of maximal elements and equilibria in linear topological spaces, Journal of Mathematical Economics 12, 233-245.

Yannelis, N.C. and W.R. Zame, 1986, Equilibria in Banach lattices without ordered preferences, Journal of Mathematical Economics 15, 85-110. 